Chapman University

Chapman University Digital Commons

Engineering Faculty Articles and Research

Fowler School of Engineering

5-10-2013

\title{
Physics of Unbounded, Broadband Absorption/Gain Efficiency in Plasmonic Nanoparticles
}

\author{
Nasim Mohammadi Estrakhri \\ Chapman University, estakhri@chapman.edu
}

Andrea Alù

The University of Texas at Austin

Follow this and additional works at: https://digitalcommons.chapman.edu/engineering_articles

\section{Recommended Citation}

N. Mohammadi Estakhri and A. Alù, "Physics of Unbounded, Broadband Absorption/Gain Efficiency in Plasmonic Nanoparticles," Physical Review B, Vol. 87, No. 20, 205418 (9 pages), May 10, 2013.

https://doi.org/10.1103/PhysRevB.87.205418.

This Article is brought to you for free and open access by the Fowler School of Engineering at Chapman University Digital Commons. It has been accepted for inclusion in Engineering Faculty Articles and Research by an authorized administrator of Chapman University Digital Commons. For more information, please contact laughtin@chapman.edu. 


\section{Physics of Unbounded, Broadband Absorption/Gain Efficiency in Plasmonic Nanoparticles}

\section{Comments}

This article was originally published in Physical Review B, volume 87, issue 20, in 2013. https://doi.org/ 10.1103/PhysRevB.87.205418

\section{Copyright}

American Physical Society 


\title{
Physics of unbounded, broadband absorption/gain efficiency in plasmonic nanoparticles
}

\author{
Nasim Mohammadi Estakhri and Andrea Alù \\ Department of Electrical and Computer Engineering, 1 University Station C0803, The University of Texas at Austin, Austin, TX 78712, USA
}

(Received 15 November 2012; published 10 May 2013)

\begin{abstract}
Anomalous resonances in properly shaped plasmonic nanostructures can in principle lead to infinite absorption/gain efficiencies over broad bandwidths of operation. By developing a closed-form analytical solution for the fields scattered by conjoined hemicylinders, we outline the fundamental physics behind these phenomena, associated with broadband adiabatic focusing of surface plasmons at the nanoscale. Over a continuous frequency range, our proposed composite nanostructure shows finite amount of absorption/amplification even in the limit of infinitesimally small intrinsic material loss/gain. Detailed physical insights are provided to justify the nature of this apparent paradox, and its counterintuitive behavior is discussed for potential applications in nonlinear optics, spasing, sensing, and energy-harvesting devices.
\end{abstract}

DOI: 10.1103/PhysRevB.87.205418

PACS number(s): 41.20.Jb, 42.25.Bs, 73.20.Mf, 78.67.Bf

\section{INTRODUCTION}

The growing interest in the optical properties of nanoparticles $^{1,2}$ has led to the discovery of many counterintuitive scattering features in plasmonic nanostructures. Due to their negative real part of permittivity, these particles support surface plasmon resonances at the nanoscale that have been proposed for many exciting applications, including field concentration, sensing, nanolasing, and optical guiding. ${ }^{3-8}$ Different configurations have been analyzed in recent years, from simple nanospheres and core-shell structures $^{6,7}$ to more complicated shapes, like crescent-shaped cylinders. ${ }^{9}$ If simple structures are known to support strong, sharp plasmon resonances, more complicated shapes may provide more complex scattering responses, such as Fano and electromagnetically-induced transparency resonances, ${ }^{10,11}$ or broadband operation. ${ }^{9}$ Including gain may further boost these effects and compensate the detrimental effects usually caused by losses. ${ }^{12,13}$ Many of the exotic properties of these geometries, however, often appear to contradict well-established physical limitations of resonant subwavelength systems, ${ }^{14}$ and the underlying physics is often difficultly captured because of the complex interaction between multiple resonances and plasmonic effects. On the other hand, a key parameter to consider in choosing a specific plasmonic geometry is the fabrication limitations dictated by technological challenges. Particles with exotic shapes and very fine features, although showing interesting electromagnetic properties, may be impractical to realize from the experimental point of view and to apply to real-life devices.

As an example that may shed new light into these phenomena, we analyze here the anomalous electromagnetic response of a rather simple composite nanoparticle, formed by two conjoined half-cylinders of arbitrary complex permittivity $\varepsilon_{1}$ and $\varepsilon_{2}$ relative to the background permittivity, and radius $a$, as shown in the inset of Fig. 1. This geometry has been recently proposed in the special configuration $\varepsilon_{1}=-\varepsilon_{2}$ to form a resonant optical nanocircuit, and previous attempts to analytically solve its scattering properties using mode-matching analysis, ${ }^{15}$ integral transformations, ${ }^{16}$ and coordinate mapping ${ }^{17}$ have led to nonphysical solutions and strong numerical instabilities. We show in the following that these challenges are associated with remarkably counterintuitive resonant phenomena, which lead to a continuous frequency range over which distributed plasmon resonances may support unbounded values of absorption or gain efficiency, i.e. finite absorption or gain even in the limit of infinitesimally small material loss/gain. By extending the analytical approach originally introduced in Ref. 17 to evaluate the polarizability of a hemicylinder, we are able to solve the complete scattering problem associated with this geometry and derive closed-form expressions for the induced fields inside and outside this composite particle. This solution provides valuable physical insights into the complex wave interaction of this particle over a broad range of frequencies, which may provide, as we discuss in the following, exciting possibilities for energy concentration, harvesting, and spasers. ${ }^{18-22}$

\section{THEORY AND FORMULATION}

\section{A. Geometry and theoretical analysis: Electrostatic solution}

We start by solving the scattering problem in the quasistatic limit, under the assumption $a<<\lambda_{0}$. An incident monochromatic wave with electric field $\mathbf{E}_{0}$ illuminates the nanostructure under an $e^{j \omega t}$ time convention, and the permittivities of the two half-cylinders can take arbitrary complex values, whose imaginary parts correspond to material loss or gain depending on their negative or positive sign. Due to symmetries and linearity, the problem may be split into two orthogonal excitations with respect to the common diameter of the structure. By using separation of variables in the 2D bipolar coordinate system, ${ }^{17}$ the potential distribution in each material may be written in integral form as

$\varphi_{i}(u, v)=\int_{0}^{\infty} U(u)\left[C_{i 1}(\lambda) \cosh (\lambda v)+C_{i 2}(\lambda) \sinh (\lambda v)\right] d \lambda$,

in which the subscript $i=1,2,0$ refers to upper, lower, and outer regions, respectively, $\lambda$ is the continuous eigenvalue, $U(u)$ is either $\cos (\lambda u)$ or $\sin (\lambda u)$ for longitudinal and transverse polarizations, respectively, and $-\infty<u<\infty$ and $-\pi<v \leqslant \pi$ are bipolar coordinate variables. The unknown coefficients $C_{i j}(\lambda)$ may be found by applying suitable boundary conditions at the various boundaries to calculate the general form of potential distribution in all space from Eq. (1). In Ref. 17, this integral expansion was used to 
determine the electric polarizability $\alpha=p / E_{0}$ of an isolated hemicylinder, where $p$ is the induced electric dipole moment, evaluated using the asymptotic expression of $\varphi_{0}$ in the far-field.
In the present case of two conjoined hemicylinders, the normalized polarizability may be analogously derived for arbitrary relative permittivity values. For longitudinal excitation, we obtain

$$
\begin{aligned}
& \alpha_{l}=\frac{\pi^{2}\left[-\varepsilon_{2}+\varepsilon_{1}\left(-1+6 \varepsilon_{2}\right)\right]+12\left(\varepsilon_{1}+\varepsilon_{2}\right)\left[\operatorname{Li}_{2}\left(\varepsilon^{-}\right)+\operatorname{Li}_{2}\left(\varepsilon^{+}\right)\right]}{1.5 \pi^{2}\left(\varepsilon_{1}+\varepsilon_{2}+2 \varepsilon_{1} \varepsilon_{2}\right)}, \\
& \varepsilon^{ \pm}=-\frac{\left(1+\varepsilon_{1}\right)\left(1+\varepsilon_{2}\right)\left(\varepsilon_{1}+\varepsilon_{2}\right)}{\varepsilon_{2} \pm \sqrt{-\left(\varepsilon_{1}-\varepsilon_{2}\right)^{2}\left(2+\varepsilon_{1}+\varepsilon_{2}\right)\left(\varepsilon_{1}+\varepsilon_{2}+2 \varepsilon_{1} \varepsilon_{2}\right)}+\varepsilon_{1}\left[1+\varepsilon_{2}\left(4+\varepsilon_{1}+\varepsilon_{2}\right)\right]},
\end{aligned}
$$

in which $\mathrm{Li}_{2}(x)$ is the polylogarithm function of second order, and analogously for the transverse excitation

$$
\alpha_{t}=\frac{\pi^{2}\left[\varepsilon_{1}+\varepsilon_{2}-6\right]-12\left(\varepsilon_{1}+\varepsilon_{2}\right)\left[\mathrm{Li}_{2}\left(\varepsilon^{-}\right)+\mathrm{Li}_{2}\left(\varepsilon^{+}\right)\right]}{1.5 \pi^{2}\left(2+\varepsilon_{1}+\varepsilon_{2}\right)} .
$$

\section{B. Absorption/gain paradox}

Having derived in closed form the polarizability of this particle, we may efficiently analyze its extinction properties as a function of the available design parameters. We start from the lossless configuration, for which all involved permittivities are purely real. Figures 1(a) and 1(b) show the calculated longitudinal polarizability for different values of $\varepsilon_{1}$ and $\varepsilon_{2}$, assuming lossless materials (real-valued $\varepsilon$ ). Since so far we have been working in the quasistatic limit, there is no radiation loss, and in the limit of no Ohmic absorption, we expect the absorbed power to be identically zero. This requires that the polarizability is purely real, as in absence of scattering loss $P_{\text {ext }}=-\omega / 2\left|E_{0}\right|^{2} \operatorname{Im}[\alpha]=P_{\mathrm{abs}}\left(P_{\mathrm{ext}}\right.$ and $P_{\mathrm{abs}}$ are extinction and absorbed powers, respectively). On the contrary, the results in Fig. 1(b) highlight continuous frequency ranges over which the polarizability has an imaginary component even in this lossless limit, consistent with some of the findings in Ref. 17 for a single hemicylinder.

To gain a better understanding of the behavior of the polarizabilities and their dependency on the permittivity and excitation, Figs. 1(c) and 1(d) also show the longitudinal and
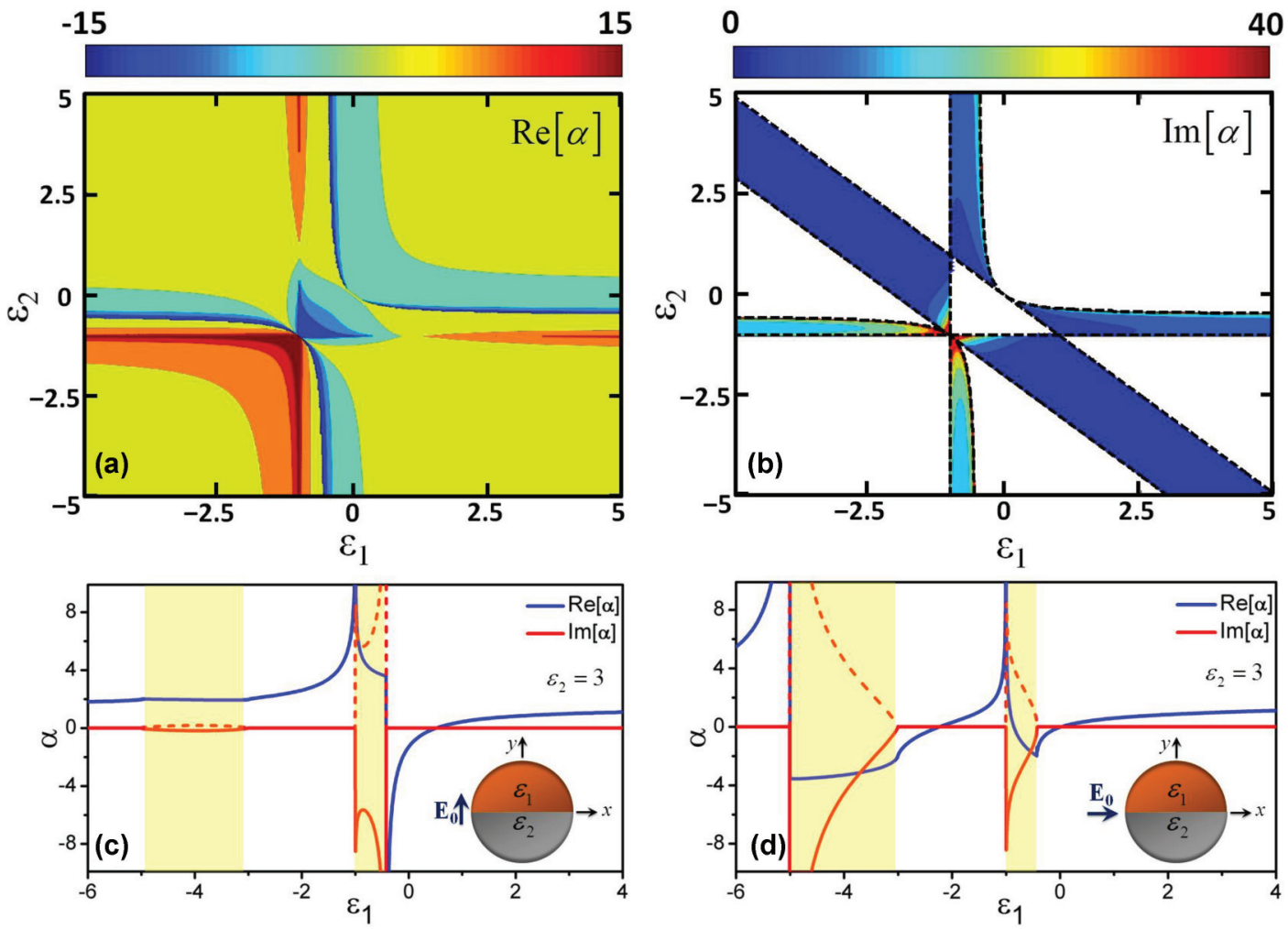

0

40

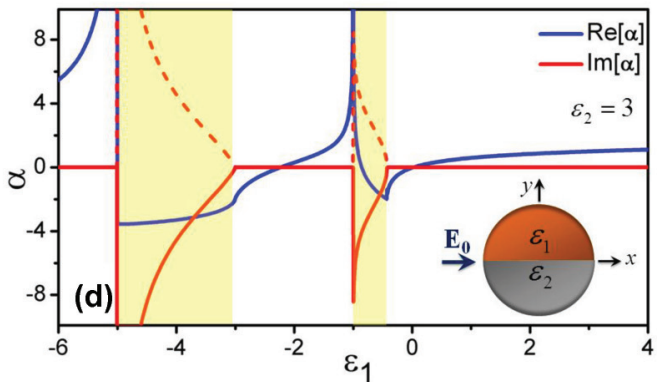

FIG. 1. (Color online) Normalized complex polarizability of two conjoined half-cylinders for different permittivity values under longitudinal excitation. (a) Real part of polarizability. (b) Imaginary part. Polarizability of the structure with $\varepsilon_{2}=3$ for (c) longitudinal and (d) transverse excitation. Particle geometry and excitation fields are shown in the inset. Shaded regions highlight the resonant ranges of this geometry, as defined in Eq. (4). 
transverse polarizabilities of the structure fixing the lower half at $\varepsilon_{2}=3$ and varying $\varepsilon_{1}$; yellow shades highlight the resonance regions in these plots. Since plasmonic properties require frequency dispersion, these plots may be also read as the variation of polarizability versus frequency, once an appropriate dispersion model for $\varepsilon_{1}$ is assumed, as discussed in the following section.

The paradoxical result illustrated in Fig. 1, for which a complex polarizability may be obtained in the static limit for lossless materials, is mathematically associated with the range of permittivities for which the arguments of $\mathrm{Li}_{2}$ have magnitude larger than one. However, the polylogarithm function $\operatorname{Li}_{N}(z)=\sum_{k=1}^{\infty} \frac{z^{k}}{k^{N}}$ is strictly convergent only for $|z|<1$, requiring that its value should be analytically continued over the whole complex plane. Two branch cuts are associated with this range of complex solutions, and complex conjugate values are admissible solutions of Eqs. (2) and (3). This implies that our geometry may be able to extract (or produce, depending on the sign of $\operatorname{Im}[\alpha]$ ) power even in the case of purely lossless (or gainless) materials. In Figs. 1(c) and 1(d), we indicate with solid (dashed) lines the solution with $\operatorname{Im}[\alpha]<0(\operatorname{Im}[\alpha]>0)$, corresponding to absorbing (amplifying) nanoparticles. ${ }^{23} \mathrm{We}$ notice that a similar response has been highlighted in the case of other plasmonic geometries involving sharp corners. ${ }^{24}$

\section{DISCUSSIONS AND PHYSICAL INSIGHTS}

In this section, we discuss the reasons behind the paradox highlighted in the previous section. We start by looking at a simple dielectric wedge structure, which models the wave interaction at the corner region shown to be at the foundation of this phenomenon. We then present novel closed-form expressions for the polarizability and potential distribution in all regions of space, which allow us to discuss the nature of the induced electric field inside and around the particle. Finally, we study the absorption properties of the proposed geometry in the electrodynamic case and investigate how realistic considerations affect these conclusions.

\section{A. Singularities and the absorption/gain paradox}

As shown in the following, the counterintuitive response of the composite particle under analysis is physically associated with the singularities induced at the two corners of the structure, which have so far been assumed as ideal mathematical edges with zero curvature at the tip. In the corner proximity, the geometry may be statically modeled as a double dielectric wedge described by the Laplace equation. Independent of the polarization of the applied field, eigensolutions may be supported by the wedge configuration for some specific values of material permittivities. ${ }^{25}$ Not surprisingly, the permittivity range over which $\operatorname{Im}[\alpha] \neq 0$ in Fig. 1(b) exactly corresponds to the quasistatic eigenresonance of a $90^{\circ}$ double dielectric wedge. It is possible to show, in fact, that the resonance of a $90^{\circ}$ double wedge arises when

$$
\begin{aligned}
& -\varepsilon_{2}-2<\varepsilon_{1}<\min \left\{-\varepsilon_{2},-1\right\}, \max \left\{-\varepsilon_{2},-1\right\}<\varepsilon_{1}<\frac{-\varepsilon_{2}}{1+2 \varepsilon_{2}} \text { for } \varepsilon_{2}>0 \\
& -\varepsilon_{2}<\varepsilon_{1}<-\frac{\varepsilon_{2}}{2 \varepsilon_{2}+1},-\varepsilon_{2}-2<\varepsilon_{1}<-1 \quad \text { for } \quad-1 / 2<\varepsilon_{2}<0 \\
& -\varepsilon_{2}<\varepsilon_{1}<\infty, \quad-\infty<\varepsilon_{1}<-\frac{\varepsilon_{2}}{2 \varepsilon_{2}+1},-\varepsilon_{2}-2<\varepsilon_{1}<-1 \text { for } \quad-1<\varepsilon_{2}<-1 / 2 \\
& -\varepsilon_{2}-2<\varepsilon_{1}<-\varepsilon_{2},-1<\varepsilon_{1}<-\frac{\varepsilon_{2}}{2 \varepsilon_{2}+1} \quad \text { for } \quad \varepsilon_{2}<-1 \text {. }
\end{aligned}
$$

These inequalities provide, in general, two/three separate continuous resonant windows of unbounded absorption/gain efficiency, defined as the ratio $\operatorname{Im}[\alpha] / \varepsilon_{i}$ with $\varepsilon_{i}$ being $\operatorname{Im}\left[\varepsilon_{1}\right]$ or $\operatorname{Im}\left[\varepsilon_{2}\right]$. In the permittivity range indicated by Eq. (4), the corners support continuous eigenmodes that are at the basis of the anomalous response discussed in the previous section.

From the physical point of view, in this resonant range a highly oscillatory potential distribution is induced around the corner of the composite nanoparticle, with strongly enhanced electric fields. In practice, this behavior is limited by nonlocal effects and the minimum corner curvature of a realistic structure. In the special case of a hemicylinder $\left(\varepsilon_{2}=1\right)$ previously studied in Refs. 15 and 17, divergent or nonphysical solutions were found in the same range. Under this condition, the two windows merge into $-3<\varepsilon_{1}<-1 / 3$, separated by a single point $\varepsilon_{1}=-1$, corresponding to the special internal resonance analyzed in Ref. 26.

In the ideal lossless limit, there is no way to distinguish between the two branch cuts, and both conjugate solutions in
Fig. 1 are equally admissible. This implies that the boundaryvalue problem is not well defined, as the uniqueness theorem does not apply to an ideal lossless scenario. ${ }^{27}$ Small losses are required to select the correct Riemann sheet and assign proper meaning to the solutions in Fig. 1. In order to address this issue, Fig. 2 shows the effect of loss/gain in $\varepsilon_{1}$ on $\operatorname{Im}[\alpha]$ for different values of $\operatorname{Re}\left[\varepsilon_{1}\right]$. Outside the resonance region, e.g. $\operatorname{Re}\left[\varepsilon_{1}\right]=-6.5$ (blue lines in Fig. 2), $\operatorname{Im}[\alpha]$ is a well-behaved continuous odd function of $\operatorname{Im}\left[\varepsilon_{1}\right]$, and it is identically zero for zero material loss. For values that lie in the continuous resonant range Eq. (4), $\operatorname{Im}[\alpha]$ is still an odd function of $\operatorname{Im}\left[\varepsilon_{1}\right]$, but it has a discontinuity at $\operatorname{Im}\left[\varepsilon_{1}\right] \rightarrow 0^{ \pm}$, associated with the ambiguity in selecting the correct Riemann sheet in the lossless case. By introducing an arbitrary amount of loss $\varepsilon_{i}<0$ or gain $\varepsilon_{i}>0$, we are able to select either the absorptive $(\operatorname{Im}[\alpha]<0)$ or emissive $(\operatorname{Im}[\alpha]>0)$ branch in Fig. 2. This implies that an arbitrarily small (but mathematically nonzero) value of loss or gain in the material can provide finite absorption or emission over a continuous bandwidth corresponding to Eq. (4), and in this 
continuous range absorption or gain efficiencies are effectively unbounded. Interestingly, smaller absorption/gain in the material can lead to larger overall absorption/gain in the nanoparticle, as the plasmonic effect at the corner is less quenched.

\section{B. Electric potential and field distributions}

Power extraction or generation can arise only in regions where the quadrature component of the potential $\operatorname{Im}\left[\varphi_{i}\right]$ is nonzero. In the quasistatic lossless limit, we would expect $\varphi_{i}$ to be exactly in phase with the excitation at all points, but in the resonant range in Eq. (4), analogous to Eq. (2), we need a nonzero imaginary component to justify power extraction. Our mathematical formalism allows calculating in closed form also the imaginary part of the potential distributions Eq. (1): by applying proper boundary conditions at all boundaries of the structure, the unknown coefficients $C_{i j}(\lambda)$ can be found for arbitrary values of $\varepsilon_{1}$ and $\varepsilon_{2}$, such as ${ }^{17}$

$$
C_{01}(\lambda)=\frac{2\left(\varepsilon_{1}-\varepsilon_{2}\right)\left(\varepsilon_{1}+\varepsilon_{2}\right)}{\varepsilon_{2}+\varepsilon_{1}\left[1+\varepsilon_{2}\left(4+\varepsilon_{1}+\varepsilon_{2}\right)\right]+\left(1+\varepsilon_{2}\right)\left(1+\varepsilon_{1}\right)\left(\varepsilon_{1}+\varepsilon_{2}\right) \cosh (\pi \lambda)}
$$

for the longitudinal polarization. Similar expressions may be found for all the other coefficients and for transverse excitation. Outside the resonant range indicated by Eq. (4), these coefficients are continuous functions of the eigenvalue $\lambda$ and may be integrated over the entire spectrum to evaluate the potential and field distributions as in Eq. (1) using a conventional numerical integration technique, i.e. the Euler method. ${ }^{28}$ In this regime, the potential and fields will be real-valued at all points in space, as expected. However, in the resonant range indicated by Eq. (4), the coefficients $C_{i j}(\lambda)$ have a simple pole in the denominator at

$$
\lambda_{p}=\frac{1}{\pi} \cosh ^{-1}\left\{-\frac{\varepsilon_{2}+\varepsilon_{1}\left[1+\varepsilon_{2}\left(4+\varepsilon_{1}+\varepsilon_{2}\right)\right]}{\left(1+\varepsilon_{2}\right)\left(1+\varepsilon_{1}\right)\left(\varepsilon_{1}+\varepsilon_{2}\right)}\right\},
$$

implying that the coefficients, which all share the same denominator, hold a nonzero residue in this range. ${ }^{29}$ In other words, each coefficient contains an integrable imaginary component at the pole location with Dirac- $\delta$ distribution sustaining the imaginary part of Eqs. (1) and (2). The amplitude of the $\delta$ distribution may be calculated in closed form by solving the residue problem as follows

$$
\begin{aligned}
\left.C_{01}(\lambda)\right|_{\lambda_{p}} & =\pi j \operatorname{Res}\left[C_{01}(\lambda), \lambda_{p}\right] \\
& =j \frac{2\left(\varepsilon_{1}-\varepsilon_{2}\right)\left(\varepsilon_{1}+\varepsilon_{2}\right)}{\left(1+\varepsilon_{2}\right)\left(1+\varepsilon_{1}\right)\left(\varepsilon_{1}+\varepsilon_{2}\right) \sinh \left(\pi \lambda_{p}\right)} \delta\left(\lambda-\lambda_{p}\right),
\end{aligned}
$$

and a similar result may be derived for all the other coefficients. Therefore, the potential distribution in Eq. (1) may be determined everywhere without ambiguity using the Cauchy's principal value integration:

$$
\begin{aligned}
\operatorname{Re}\left[\varphi_{i}(u, v)\right]= & \mathrm{p} \cdot \mathrm{v} \cdot \int_{0}^{\infty} U(u)\left[C_{i 1}(\lambda) \cosh (\lambda v)\right. \\
& \left.+C_{i 2}(\lambda) \sinh (\lambda v)\right] d \lambda \\
\operatorname{Im}\left[\varphi_{i}(u, v)\right]= & \left.U(u)\right|_{\lambda_{p}}\left[C_{i 1}\left(\lambda_{p}\right) \cosh \left(\lambda_{p} v\right)\right. \\
& \left.+C_{i 2}\left(\lambda_{p}\right) \sinh \left(\lambda_{p} v\right)\right],
\end{aligned}
$$

leading to a closed-form expression for the imaginary part of potential and field distribution. As an example in the case of conjoined hemicylinders and longitudinal excitation, the imaginary part of the potential distribution in the upper halfcylinder may be written in closed-form as

$$
\begin{aligned}
\operatorname{Im}\left[\varphi_{1}(u, v)\right]= & \frac{2 E_{0} \cos \left(\lambda_{p} u\right)}{\left(\varepsilon_{1}+1\right)\left(\varepsilon_{2}+1\right)\left(\varepsilon_{1}+\varepsilon_{2}\right) \sinh \left(\lambda_{p} \pi\right)}\left\{\left[-\left(\varepsilon_{1}-1\right)\left(\varepsilon_{2}+1\right)\left(\varepsilon_{1}+\varepsilon_{2}\right) \operatorname{coth}\left(\lambda_{p} \pi / 2\right)+\frac{\left(\varepsilon_{1}-\varepsilon_{2}\right)^{2}}{\sinh \left(\lambda_{p} \pi\right)}\right]\right. \\
& \left.\times \sinh \left[\lambda_{p}(\pi-v)\right]+2\left(\varepsilon_{2}-\varepsilon_{1}\right) \frac{\sinh \left[\lambda_{p}(\pi / 2-v)\right]}{\sinh \left(\lambda_{p} \pi / 2\right)}\right\}
\end{aligned}
$$

We recall that this component of the potential is responsible for absorption/gain and can therefore provide interesting insights into the apparent paradox outlined in the previous section. Analogous expressions may be derived for the potential distribution at every point in space. It is quite remarkable that, in this geometry, we are able to derive in closed form the imaginary component of the potential distribution everywhere in space. Similarly, we can write the imaginary part of the polarizabilities given by Eqs. (2) and (3) in a simple closed form using direct integration of the singularity in the integrand:

$$
\begin{aligned}
& \operatorname{Im}\left[\alpha_{l}\right]=8 \lambda_{p} \operatorname{sign}\left[\frac{\left(\varepsilon_{1}+\varepsilon_{2}\right)}{\varepsilon_{1}+\varepsilon_{2}+2}\right] \frac{\left(\varepsilon_{1}+\varepsilon_{2}\right)\left(\varepsilon_{1} \varepsilon_{2}-1\right) \operatorname{coth}\left(\lambda_{p} \pi / 2\right) \sinh \left(\lambda_{p} \pi\right)-\left(\varepsilon_{1}-\varepsilon_{2}\right)^{2}}{\left(\varepsilon_{1}+1\right)\left(\varepsilon_{2}+1\right)\left(\varepsilon_{1}+\varepsilon_{2}\right) \sinh ^{2}\left(\lambda_{p} \pi\right)} \\
& \operatorname{Im}\left[\alpha_{t}\right]=8 \lambda_{p} \operatorname{sign}\left[\frac{\left(\varepsilon_{1}+\varepsilon_{2}\right)}{\varepsilon_{1}+\varepsilon_{2}+2}\right] \frac{\left(\varepsilon_{1}+\varepsilon_{2}\right)\left(\varepsilon_{1} \varepsilon_{2}-1\right) \cosh \left(\lambda_{p} \pi\right)+\varepsilon_{1} \varepsilon_{2}\left(\varepsilon_{1}+\varepsilon_{2}-2\right)+\varepsilon_{2}^{2}+\varepsilon_{1}^{2}-\varepsilon_{1}-\varepsilon_{2}}{\left(\varepsilon_{1}+1\right)\left(\varepsilon_{2}+1\right)\left(\varepsilon_{1}+\varepsilon_{2}\right) \sinh ^{2}\left(\lambda_{p} \pi\right)},
\end{aligned}
$$



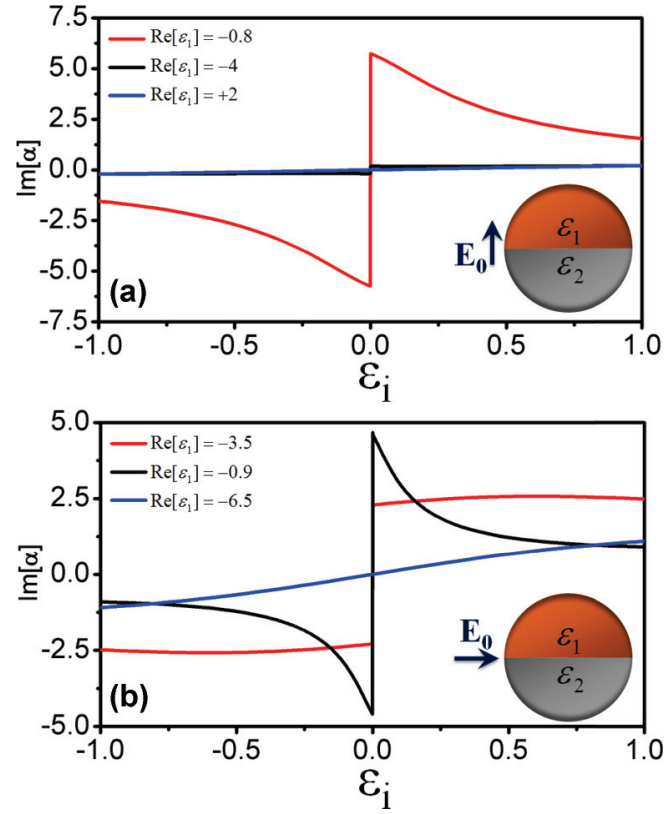

FIG. 2. (Color online) Imaginary part of polarizability versus $\operatorname{Im}\left[\varepsilon_{1}\right]$ for different values of $\operatorname{Re}\left[\varepsilon_{1}\right]$ and $\varepsilon_{2}=3$ for (a) longitudinal and (b) transverse illumination. Particle geometry and excitation fields are shown in the inset.

which allows calculating $P_{\text {ext }}=-\omega / 2\left|E_{0}\right|^{2} \operatorname{Im}[\alpha]=P_{\mathrm{abs}}$ in closed form. These expressions are consistent with Eqs. (2) and (3) and are clearly valid only in the resonant range given by Eq. (4) and zero elsewhere. The sign term in this last equation ensures the proper choice of the branch cut in the lossless limit. By adding an infinitesimally small amount of loss/gain, the solution will automatically collapse to the correct branch, consistent with Fig. 2.

Figures 3(a) and 3(b), as an example, show the real and imaginary parts of the potential distribution for a hemicylinder $\left(\varepsilon_{2}=1\right)$ with $\varepsilon_{1}=-1.1$ and longitudinal excitation. The imaginary part is calculated using our closed-form expressions, whereas the real part is obtained by numerical integration of Eq. (1). The imaginary component of the potential essentially represents an eigenmode of the structure, in quadrature with the impinging field and supported by plasmonic resonances at the two corners, with an amplitude linked to the value of excitation. This distribution, integrated over the nanoparticle volume, effectively sustains the extracted/generated power. Our analytical solution ensures that, in the corner proximity, the potential varies in the form $\rho^{v}$, in which $v$ is purely imaginary inside the resonance region, forming a highly oscillatory distribution analogous to Figs. 3(a) and 3(b) around these points. It should be noted that, in the ideal lossless limit, this distribution is not square-integrable, as it leads to a finite value of extracted/generated power for $\varepsilon_{i} \rightarrow 0 . .^{30}$ This finding, consistent with the unbounded energy density found near sharp corners in other geometries, ${ }^{31}$ explains the reason behind the nonuniqueness of our solution in the lossless limit. Figures 3(c) and 3(d) show the corresponding field distributions in the same structure, calculated analytically as $\mathbf{E}=-\nabla \varphi$. Plasmonic oscillations around the corners [Figs. 3(a) and 3(b)] result in enhanced fields, which may become infinite at the edge point in the lossless case for an ideal corner.

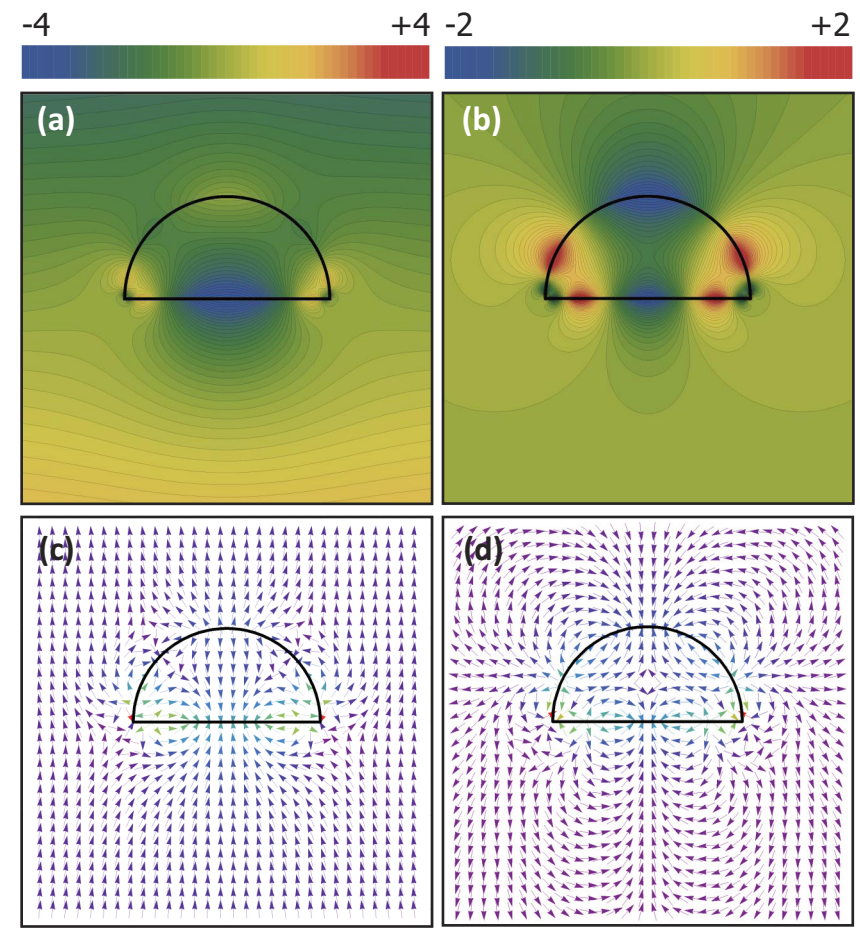

FIG. 3. (Color online) (a) Real part and (b) imaginary part of the potential distribution for a half-cylinder with permittivity $\varepsilon_{1}=-1.1$ under longitudinal excitation, normalized to the impinging potential amplitude; (c) real and (d) imaginary parts of the field distribution in the particle.

Inspecting the imaginary part of the potential distribution in Fig. 3(b), we indeed notice strong plasmonic oscillations around the nanoparticle corners. The variation of potential along the particle diameter is plotted in Fig. 4(a), highlighting that the surface plasmon supported by the metal-dielectric interface is adiabatically focused towards the corners, with a finer and finer spatial variation as the corner is approached. This effect, supported over the whole resonant range indicated by Eq. (4), produces broadband, largely enhanced electric fields, and it sustains absorption/amplification even for infinitesimally small values of material loss/gain. Essentially, the surface plasmon is adiabatically focused towards the corner, as if it were traveling to infinity (inset of Fig. 4), explaining the reason why negligible losses (gain) are sufficient to sustain large absorption (amplification). Different from conventional adiabatic focusing of surface plasmons, in this geometry, this effect is achieved at the nanoscale.

Figure 4(b) shows the potential variation along the common diameter of the particle for a different example $\left(\varepsilon_{1}=-2, \varepsilon_{2}=\right.$ 1). The different behavior between $\varepsilon_{1}=-1.1$ and $\varepsilon_{1}=-2$ can be interestingly explained considering the wedge solution. For values of $\varepsilon_{1}$ near -1 , the frequency of spatial oscillations is much larger compared to $\varepsilon_{1}=-2$, resulting in oscillations extended farther from the corners. For these situations, the field enhancement may be extended more broadly all over the particle, with interesting possibilities to more effectively enhance optical nonlinearities. These distributed resonances and adiabatic focusing have direct analogies with the resonant distribution highlighted in Refs. 9, 32, and 33 for crescent-shaped 

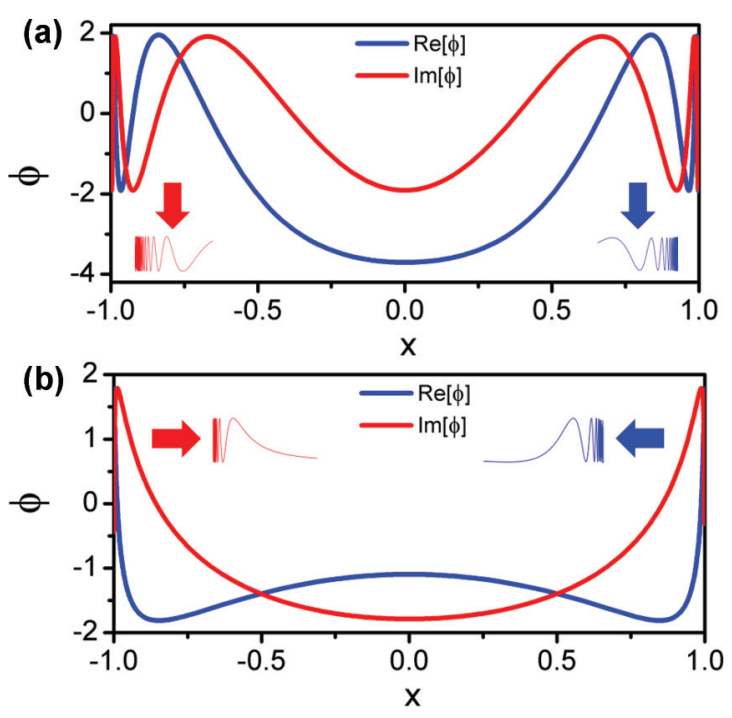

FIG. 4. (Color online) (a) Real (blue) and imaginary (red) parts of the normalized potential distribution for a half-cylinder with $\varepsilon_{1}=-1.1$ under longitudinal excitation along the $x$ axis. (b) Same distributions when $\varepsilon_{1}=-2$. Closer views of the calculated potential around the corner points are shown in inset.

and touching plasmonic cylinders, but it is obtained here in an arguably simpler geometry over a flat surface and controllable frequency bands.

\section{Radiation losses and absorption cross-section}

The previous analysis highlights that the apparent paradox of unbounded absorption/gain efficiencies in the proposed nanoparticle is related to two relevant assumptions: ideal singularities in the nanoparticle geometry (perfect corners) and quasistatic solution. In the following, we relax both these assumptions and analyze how these effects may be translated into realistic geometries and setups. In the long-wavelength limit, as long as the dipolar contribution dominates the scattering response, the quasistatic solution can be easily extended to the dynamic regime to include effects of radiation and retardation. ${ }^{34}$ The dynamic Mie dipolar coefficient $C_{1}$ is related to the static polarizabilities given by Eqs. (2) and (3) via $C_{1}=\left(-1+j 8 x_{0}^{-2} \alpha^{-1} / \pi\right)^{-1}, x_{0}=k_{0} a$, which includes now radiation losses. This procedure is consistent with the fact that, in the long-wavelength limit, the second-order correction to the polarizability response is due to dipolar radiation, taken into account by the additional imaginary term.

Figure 5 shows the absorption cross-section normalized to the physical width of the particle for composite cylinders with $2 a=40 \mathrm{~nm}$, compared to the case of a homogeneous cylinder of same size. In this case, in order to include also frequency dispersion and realistic material absorption, the upper halfcylinder is chosen to be silver with $\varepsilon_{r}=\varepsilon_{\infty}-\omega_{p}^{2} / \omega(\omega-j \Gamma)$, $\varepsilon_{\infty}=5, \omega_{p}=2 \pi \times 2175 \mathrm{THz}$, and $\Gamma=2 \pi \times 4.35 \mathrm{THz} .{ }^{35}$ We compare the case of a silver hemicylinder $\left(\varepsilon_{2}=1\right)$ and the case $\varepsilon_{2}=3$, which have different resonant bands following Eq. (4). The results confirm that absorption/gain may be largely enhanced over a continuous and controllable frequency band, significantly broadening the range and level of absorption/gain compared to a full circular rod of the same material. For
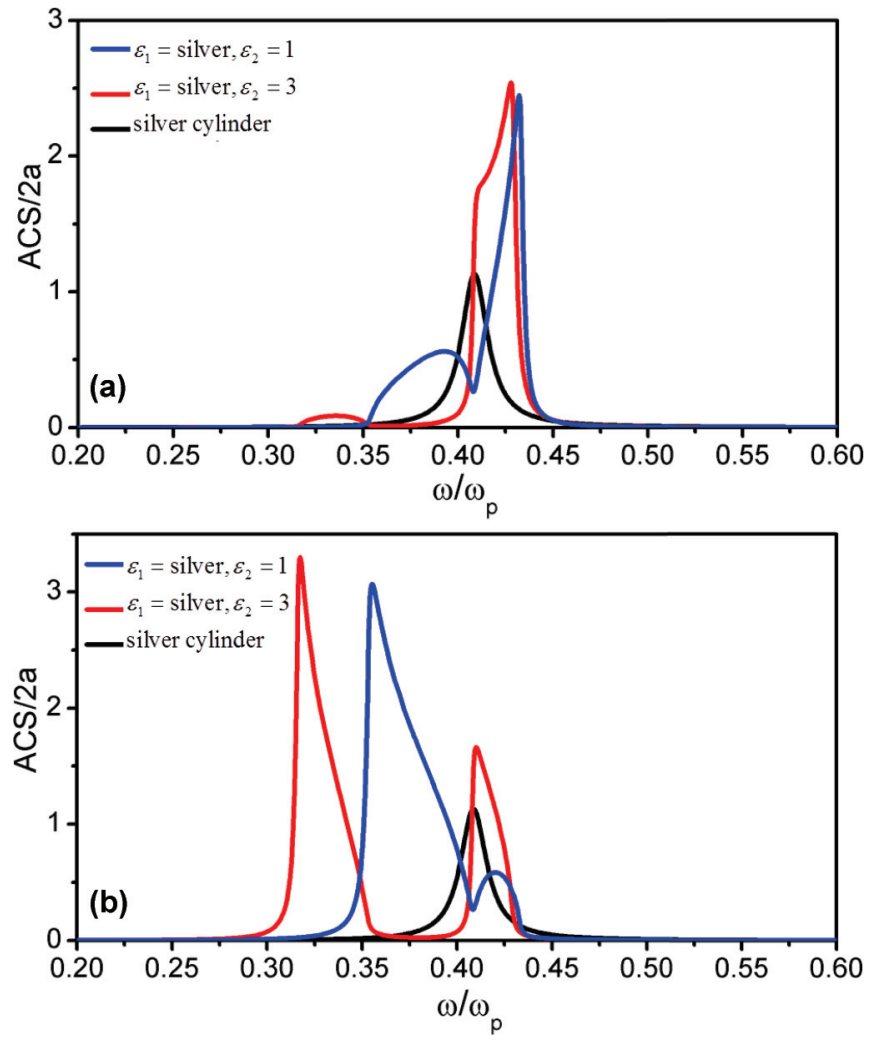

FIG. 5. (Color online) Normalized absorption cross-section for (a) longitudinal and (b) transverse excitation of a composite nanoparticle with upper half-cylinder made of silver and different values of $\varepsilon_{2}$. The full cylinder case is also shown for comparison.

a half-cylinder, the absorption is drastically enhanced in the frequency bands corresponding to the resonance region $\left(-3<\varepsilon_{1}<-1\right.$ and $\left.-1<\varepsilon_{1}<-1 / 3\right)$ and is negligible at other frequencies. We observe that this particle shows a lower amount of absorption around the frequency for which $\varepsilon_{1}=-1$, at which we actually have the highest absorption in the full cylinder case, consistent with the previous analysis. Quite counterintuitively, this absorption band does not rely on material losses and in fact is larger in the limit of zero losses, as discussed in Fig. 2. Another example of this phenomenon, although much more limited in bandwidth, is evident in transition metamaterials. ${ }^{36}$ Compared to a full cylinder of the same material, the absorption is drastically enhanced and its bandwidth significantly broadened. Since we can control the resonance range with $\varepsilon_{2}$ following Eq. (4), the structure can be designed to show high absorption efficiencies in two separate bands over the desired frequency ranges.

In order to gain further insight into the effect of material and radiation losses on the resonance behavior of the structure, we also separately study these effects in a golddielectric configuration. We consider conjoined half-cylinders with diameter $2 a=40 \mathrm{~nm}$, in which now the upper half is made of gold following a Drude model with $\varepsilon_{\infty}=1.53$, $\omega_{p}=2 \pi \times 2069 \mathrm{THz}$, and $\Gamma=2 \pi \times 17.64 \mathrm{THz}$ based on experimental measurement data. ${ }^{35}$ Again, three configurations are studied separately: $\varepsilon_{2}=1, \varepsilon_{2}=3$, and a full gold cylinder for comparison. Figure 6 shows the normalized absorption cross-section versus frequency for three different scenarios: 

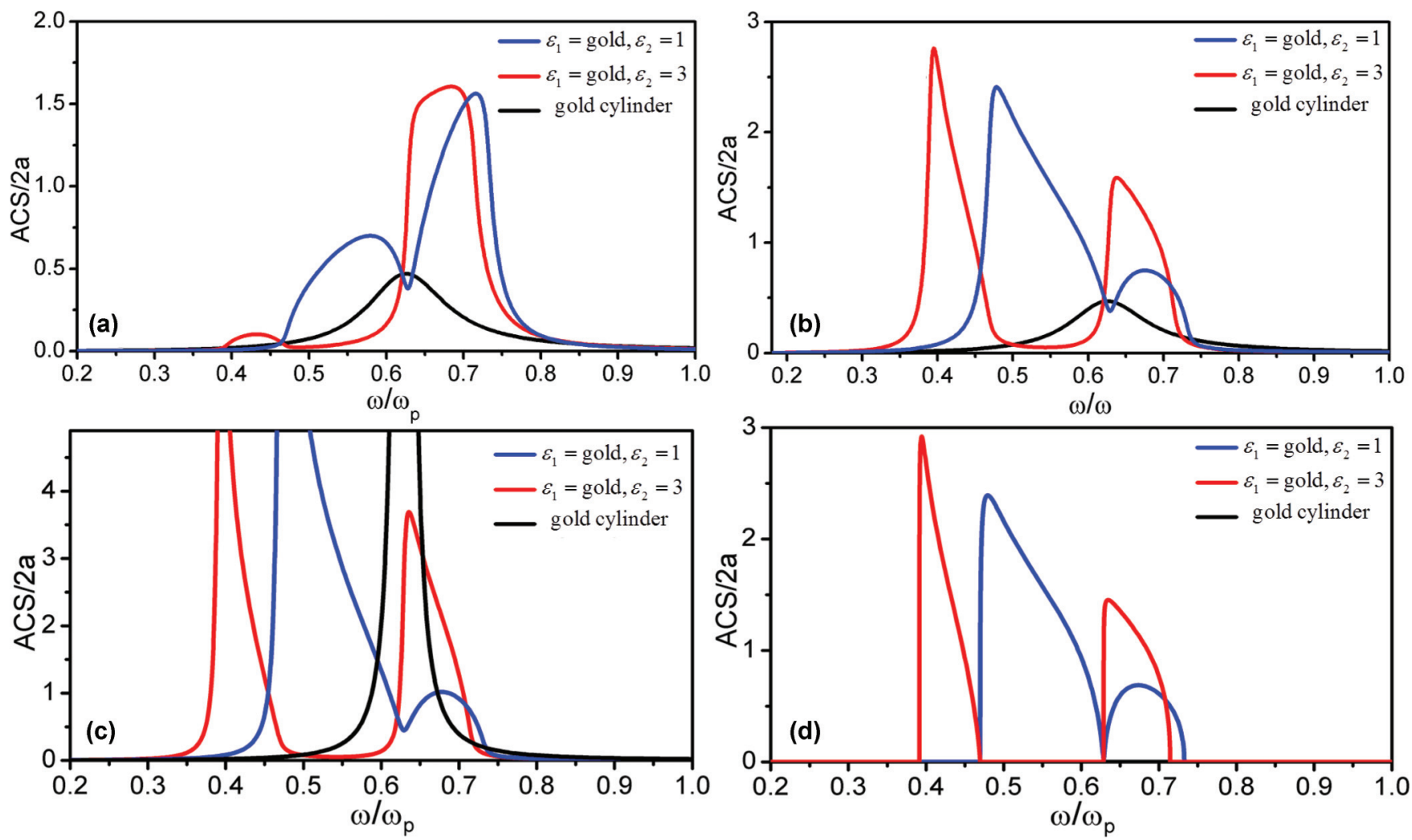

FIG. 6. (Color online) Normalized absorption cross-section for different configurations: the upper half-cylinder is gold, while the lower part is either $\varepsilon_{2}=1$ (blue curves) or $\varepsilon_{2}=3$ (red curves). A full cylinder composed of gold (black curves) is also included for comparison. (a) Longitudinal excitation. (b) Transverse polarization. (c) Normalized absorption cross-section for transverse excitation under the quasistatic approximation; (d) same as (b), but neglecting gold losses.

in the first two panels, we consider both realistic losses and retardation effects for different polarization of the impinging wave. Panel (c) shows the absorption for transverse excitation neglecting retardation but including realistic losses. Panel (d) on the other hand includes the retardation effect but assumes $\Gamma=0$ (lossless gold).

Compared to silver (Fig. 5), gold provides slightly lower absorption due to damping of the plasmonic resonance near the corners in the presence of a larger material and radiation losses. This can be explained also inspecting Fig. 6(d), in which we totally neglect material loss. In general, with conventional lowloss plasmonic materials (e.g. silver and gold), the focusing effect still dominates the absorption features of these particles. The effect of retardation can be observed in Fig. 6(c). By including scattering loss, as expected, the absorption crosssection is broadened and dampened. It is interesting that, in the case of a single full cylinder, scattering loss affects the total absorption much more drastically than in the composite configurations.

\section{Realistic configurations}

In order to demonstrate the realistic applicability of the proposed structure, we analyze now the effect of finite curvature at the corners. As discussed in Ref. 31, when a mathematical edge is replaced by one with nonzero curvature, the continuous eigenresonance range is necessarily converted into a set of discrete resonance frequencies, which ensures that Chu's fundamental limit is satisfied. ${ }^{14}$ The amount of realizable absorption will depend on how adiabatically surface plasmon resonances may be focused and absorbed before the edge is terminated. We used full-wave simulations to study this effect for different curvature values. Absorption cross-section of a blunted hemicylinder with permittivity $\varepsilon_{1}=-0.529-j \varepsilon_{i}$ and $2 a=40 \mathrm{~nm}$ is compared in Fig. 7 to an ideal geometry with the same parameters. The full cylinder case is also shown for comparison. We notice that the absorption phenomenon is pretty robust for finite values

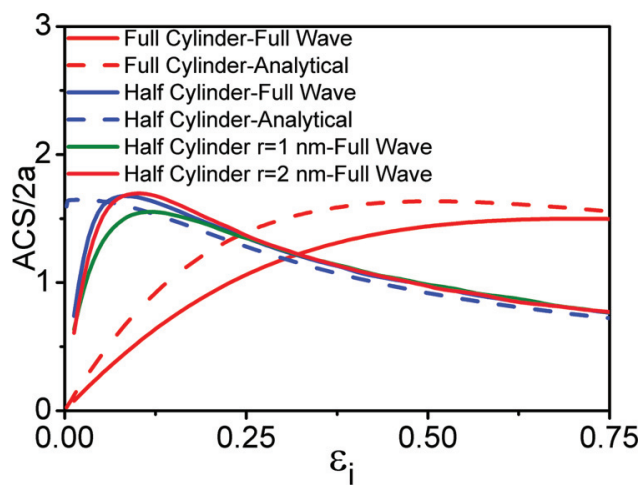

FIG. 7. (Color online) Normalized absorption cross-section versus material loss for a hemicylinder with $\varepsilon_{1}=-0.529-j \varepsilon_{i}$ compared to full-wave simulations for 1- and 2-nm curvature radii. The full cylinder is also shown for comparison. 


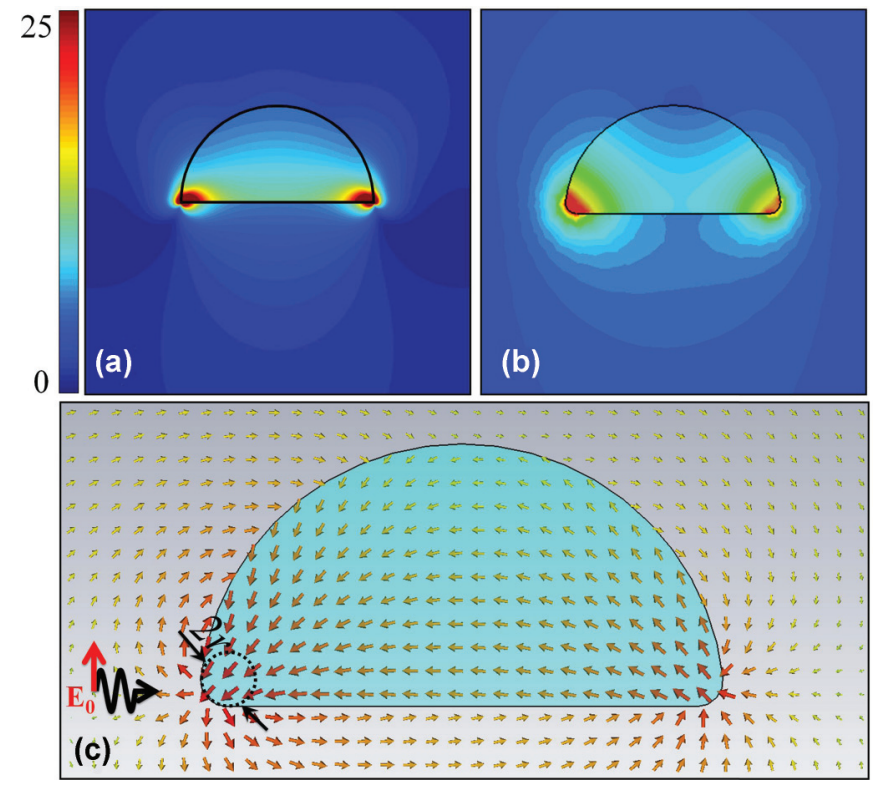

FIG. 8. (Color online) Amplitude of the electric field distribution for (a) an ideal silver half-cylinder with $\varepsilon_{1}=-0.529-0.026 j$, calculated with our analytical formulation, and (b) a blunted configuration at the corresponding frequency. (c) Power flow inside and outside the geometry under monochromatic plane wave excitation for the blunted configuration.

of material loss, and the edge bluntness effectively sets the lower level of $\left|\varepsilon_{i}\right|$ for which large absorption/gain may be achieved. In other words, when considering corners with finite curvature, absorption/gain efficiencies are inherently bounded and fundamentally limited by how sharp (relative to the radius of the particle), the corner may be made. Similar results are found in the case of the singular geometry simulated with finite-integration methods (blue curve), as a finite curvature is automatically introduced by numerical meshing. Our results show that significantly large and broadband absorption/gain effects may be achieved with realistic nanoparticle geometries.

Our full-wave simulations confirm the robustness of this phenomenon on the corner curvature and edge bluntness, consistent with previous results for other types of plasmonic resonances. ${ }^{37}$ For sharper edges, the number of quantized resonances increases, and the overall effect gets closer to the ideal solution. ${ }^{38}$ Figures $8(a)$ and $8($ b) show the field distribution for a silver half-cylinder having an ideal corner using our analytical solution in the quasistatic limit versus a blunted structure with $2 a=40 \mathrm{~nm}$ and radius of curvature $r=2 \mathrm{~nm}$ using full-wave simulations at $f=925 \mathrm{THz}$ for longitudinal excitation. Figure 8(c) also shows the power flow in the blunted structure. Interestingly, even with a relatively large edge curvature, and including scattering losses and dynamic effects, highly oscillatory fields are still induced around the corners, and field enhancement is pretty comparable with the ideal case. The small asymmetry in the distribution is due to the direction of the impinging wave, but since the particle is small compared to wavelength, this effect is almost negligible. Power flow is plotted in a $\log _{100}$ scale, implying large power concentration inside the particle, responsible for large absorption efficiencies. In other words, under the resonance condition, power is strongly concentrated inside the particle, giving rise to very large absorption regardless of the small amount of material loss.

\section{E. Potential applications}

The distributed resonances and anomalous behavior of the proposed composite nanoparticle may have many exciting applications, including enhanced energy harvesting and spasers $^{12,18}$ based on materials with limited absorption/gain coefficients and an arguably simple configuration from the fabrication point of view. These resonances may be broadband and with a bandwidth and enhancement level controllable by geometry and design. In this sense, we notice that the bandwidth of enhancement is effectively controlled by the corner geometry, and sharper corners can support eigenmodes over even broader continuous bandwidths. We are currently exploring related geometries, such as cylindrical and spherical caps, for which anomalous, broadband enhancement of absorption and gain may be achieved over even larger frequency ranges, based on similar principles. Adiabatic plasmonic focusing at the corners may also be used for other exciting applications such as enhanced optical nonlinear effects, e.g. switching and nanomemories. ${ }^{11}$ The field enhancement may be tailored to be extended all over the particle volume or be confined only around the corners, with exciting implications for these applications (Fig. 4). The rapid and sharp variation of absorption versus frequency observed in Figs. 5 and 6 can also be used for sensing ${ }^{39}$ with sharp line widths that are comparable to the ones associated with Fano phenomena. ${ }^{10}$ Finally, these effects may have a great interest in boosting the usually low values of gain coefficients in natural optical materials, of great interest for loss compensation in metamaterials and plasmonics, ${ }^{13,40-42}$ as well as for efficient spasers. ${ }^{12,18,19,43}$

\section{CONCLUSIONS}

We have analyzed here the scattering boundary-value problem of two conjoined subwavelength half-cylinders and analyzed its drastically enhanced absorption properties. We have shown that, in the ideal case of perfect corners, this geometry may provide broadband light absorption or amplification in the limit of negligible material loss or gain, respectively. This absorption paradox has been shown to be associated with the singularities in the geometry and the adiabatic focusing of broadband surface plasmons supported at the corners. A closed-form solution was derived for the scattering and absorption properties of the composite nanostructure, and simple conditions on the material permittivities have been derived to control the position of the absorption band. Energy harvesting, sensing, broadband lasing, and boosting of nonlinearities have been discussed as potential applications of this nanodevice, also in relation to its robustness to realistic level of losses/gain in plasmonic materials and finite curvature of the proposed geometry.

\section{ACKNOWLEDGMENTS}

This work has been supported by the AFOSR YIP Award No. FA9550-11-1-0009 and the ONR MURI Grant No. N00014-10-1-0942. 
*Corresponding author: alu@mail.utexas.edu

${ }^{1}$ Y. Sun and Y. Xia, Science 298, 2176 (2002).

${ }^{2}$ V. Bastys, I. Pastoriza-Santos, B. Rodriguez-Gonzalez, R. Vaisnoras, and L. M. Liz-Marzan, Adv. Funct. Mater. 16, 766 (2006).

${ }^{3}$ W. L. Barnes, A. Dereux, and T. W. Ebbesen, Nature 424, 824 (2003).

${ }^{4}$ S.A. Maier, Plasmonics: Fundamentals and Applications (Springer, New York, 2007).

${ }^{5}$ A. Polman, Science 322, 868 (2008).

${ }^{6}$ K. L. Kelly, E. Coronado, L. Zhao, and G. C. Schatz, J. Phys. Chem. B 107, 668 (2003).

${ }^{7}$ E. Prodan, C. Radloff, N. J. Halas, and P. Nordlander, Science 302, 419 (2003).

${ }^{8}$ T. Klar, M. Perner, S. Grosse, G. von Plessen, W. Spirkl, and J. Feldmann, Phys. Rev. Lett. 80, 4249 (1998).

${ }^{9}$ A. Aubry, D. Y. Lei, S. A. Maier, and J. B. Pendry, Phys. Rev. B 82, 125430 (2010).

${ }^{10}$ B. Luk'yanchuk, N. I. Zheludev, S. A. Maier, N. J. Halas, P. Nordlander, H. Giessen, and C. Chong, Nat. Mater. 9, 707 (2010).

${ }^{11}$ C. Argyropoulos, P.-Y. Chen, F. Monticone, G. D'Aguanno, and A. Alù, Phys. Rev. Lett. 108, 263905 (2012).

${ }^{12}$ N. I. Zheludev, S. L. Prosvirnin, N. Papasimakis, and V. A. Fedotov, Nature Photon. 2, 351 (2008).

${ }^{13}$ M. I. Stockman, Phys. Rev. Lett. 106, 156802 (2011).

${ }^{14}$ L. J. Chu, J. Appl. Phys. 19, 1163 (1948).

${ }^{15}$ H. Kettunen, H. Wallen, and A. Sihvola, J. Electrost. 67, 890 (2009).

${ }^{16}$ A. Salandrino, A. Alù, and N. Engheta, J. Opt. Soc. Am. B 24, 3007 (2007).

${ }^{17}$ M. Pitkonen, J. Electromagn. Waves Appl. 24, 1267 (2010).

${ }^{18}$ D. J. Bergman and M. I. Stockman, Phys. Rev. Lett. 90, 027402 (2003).

${ }^{19}$ M. A. Noginov, G. Zhu, A. M. Belgrave, R. Bakker, V. M. Shalaev, E. E. Narimanov, S. Stout, E. Herz, T. Suteewong, and U. Wiesner, Nature 460, 1110 (2009).

${ }^{20}$ E. S. Andrianov, A. A. Pukhov, A. V. Dorofeenko, A. P. Vinogradov, and A. A. Lisyansky, Opt. Lett. 36, 4302 (2011).

${ }^{21}$ E. S. Andrianov, A. A. Pukhov, A. V. Dorofeenko, and A. P. Vinogradov, J. Commun. Technol. Electron. 57, 106 (2012).

${ }^{22}$ M. W. Tsai, T. H. Chuang, C. Y. Meng, Y. T. Chang, and S. C. Lee, Appl. Phys. Lett. 89, 173116 (2006).

${ }^{23}$ We notice that the author of Ref. 17 arbitrarily selected one of the two admissible branches, choosing opposite signs of the imaginary part in the different ranges with complex polarizability. Mathematically, there is no reason to choose one branch or the other, as both solutions satisfy all boundary conditions of the system. By choosing opposite signs, we imply that the particle absorbs and emits in the two different permittivity ranges.

${ }^{24} \mathrm{G}$. W. Milton, The Theory of Composites, Cambridge Monographs on Applied and Computational Mathematics (Cambridge University Press, Cambridge, 2002)

${ }^{25}$ M. G. Silveirinha and N. Engheta, Phys. Rev. B 76, 245109 (2007).

${ }^{26}$ A. Alù and N. Engheta, New J. Phys. 11, 013026 (2009).

${ }^{27}$ R. F. Harrington, Time-Harmonic Electromagnetic Fields (McGraw-Hill, New York, 1961).

${ }^{28}$ M. N. O. Sadiku, Numerical Techniques in Electromagnetics (CRC, Boca Raton, FL, 2001).

${ }^{29} \mathrm{~J}$. W. Brown and R. V. Churchill, Complex Variables and Applications (McGraw-Hill, New York, 1996), Vol. 7.

${ }^{30}$ J. Helsing, R. C. McPhedran, and G. W. Milton, New J. Phys. 13, 115005 (2011)

${ }^{31}$ L.C. Davis, Phys. Rev. B 14, 5523 (1976).

${ }^{32}$ R. McPhedran and W. Perrins, Appl. Phys. A 24, 311 (1981).

${ }^{33}$ A. Aubry, D. Y. Lei, S. A. Maier, and J. B. Pendry, Phys. Rev. Lett. 105, 233901 (2010).

${ }^{34}$ J. E. Sipe and J. V. Kranendonk, Phys. Rev. A 9, 1806 (1974).

${ }^{35}$ M. A. Ordal, R. J. Bell, R. W. Alexander, Jr., L. L. Long, and M. R. Querry, Appl. Opt. 24, 4493 (1985).

${ }^{36}$ N. M. Litchinitser, A. I. Maimistov, I. R. Gabitov, R. Z. Sagdeev, and V. M. Shalaev, Opt. Lett. 33, 2350 (2008).

${ }^{37}$ Y. Luo, D. Y. Lei, S. A. Maier, and J. B. Pendry, Phys. Rev. Lett. 108, 023901 (2012).

${ }^{38}$ R. Fuchs, Phys. Rev. B 11, 1732 (1975).

${ }^{39}$ N. Liu, M. Mesch, T. Weiss, M. Hentschel, and H. Giessen, Nano Lett. 10, 2342 (2010).

${ }^{40}$ S. Xiao, V. P. Drachev, A. V. Kildishev, X. Ni, U. K. Chettiar, H.-K. Yuan, and V. M. Shalaev, Nature (London) 466, 735 (2010).

${ }^{41}$ M. A. Noginov, G. Zhu, M. Bahoura, J. Adegoke, C. E. Small, B. A. Ritzo, V. P. Drachev, and V. M. Shalaev, Opt. Lett. 31, 3022 (2006).

${ }^{42}$ S. A. Ramakrishna and J. B. Pendry, Phys. Rev. B 67, 201101(R) (2003).

${ }^{43}$ Y. J. Lu, J. Kim, H. Y. Chen, C. Wu, N. Dabidian, C. E. Sanders, C. Y. Wang, M. Y. Lu, B. H. Li, X. Qiu, W. H. Chang, L. J. Chen, G. Shvets, C. K. Shih, and S. Gwo, Science 337, 450 (2012). 